\title{
Predictive Value of Bronchoalveolar Lavage in Excluding a Diagnosis of Pneumocystis carinii Pneumonia During Prophylaxis with Aerosolized Pentamidine
}

\author{
Adrian U. Teuscher, ${ }^{*}$ Milos Opravil, Robert Theiler, \\ Ruedi Speich, Herbert Kuster, Walter Siegenthaler, \\ Erich W. Russi, and Ruedi Lüthy
}

From the Department of Medicine, University Hospital, Zürich, Switzerland

\begin{abstract}
We assessed the negative predictive value of bronchoalveolar lavage (BAL) for Pneumocystis carinii pneumonia (PCP) during prophylaxis with aerosolized pentamidine. On the basis of the assumption that undiagnosed and untreated PCP would progress and become clinically apparent, for 3 months we prospectively followed 34 consecutive cases in which BAL had not detected PCP. All patients were immunodeficient, had a symptomatic human immunodeficiency virus infection, and were evaluated for possible PCP during prophylaxis with aerosolized pentamidine. No transbronchial biopsies were performed. In 32 of 34 cases, a diagnosis of PCP could be excluded because of other definite diagnoses or improvement during the follow-up. Despite negative results of an examination of their BAL fluid, two patients received empirical treatment that was active against PCP; these patients were regarded as possibly having undiagnosed PCP. Thus, the negative predictive value of BAL alone was at least $94 \%$ ( 32 of 34 cases) in excluding a diagnosis of PCP during prophylaxis with aerosolized pentamidine.
\end{abstract}

Although aerosolized pentamidine has been shown to be effective for primary and secondary prevention of Pneumocystis carinii pneumonia (PCP) in patients infected with human immunodeficiency virus (HIV) [1-3], breakthrough PCP occurs despite prophylaxis in $8 \%-22 \%$ of these patients per year [2,3]. A delay in diagnosis in these cases worsens the prognosis, as mortality due to PCP is associated with advanced disease and respiratory failure [4]. When no $P$. carinii is detected in spontaneous or induced sputum, fiberoptic bronchoscopy with bronchoalveolar lavage (BAL) is the diagnostic procedure of choice; it is well tolerated and has a sensitivity of between $86 \%$ and $100 \%$ [5-7]. Transbronchial biopsy is associated with a higher risk of complications and does not substantially improve the overall yield in detection of $P$. carinii in patients who have received no prophylaxis [5, 7]. Therefore, it has not been used in our institution as an initial procedure. However, a decrease in the diagnostic yield of BAL was recently reported in regard to patients receiving pentamidine prophylaxis, a finding that suggests the need for performance of transbronchial biopsy during bronchoscopy as well $[7,8]$. We examined whether a diagnosis of PCP was missed during evaluation of high-risk patients who were re-

Received 3 June 1992; revised 4 November 1992.

* Current address: University of Minnesota, Diabetes Center, Minneapolis, Minnesota 55455.

Reprints or correspondence: Dr. Ruedi Lüthy, Division of Infectious Diseases, Department of Medicine, University Hospital, 8091 Zürich, Switzerland.

Clinical Infectious Diseases 1993;16:519-22

(C) 1993 by The University of Chicago. All rights reserved. 1058-4838/93/1604-0011\$02.00 ceiving pentamidine prophylaxis and in whom no $P$. carinii had been detected by BAL and no transbronchial biopsy had been performed. The clinical outcome was prospectively analyzed on the basis of the assumption that undiagnosed and untreated PCP would progress and become clinically apparent during a 3-month follow-up.

\section{Methods}

Between September 1989 and March 1991 at our facility, we studied all patients with HIV infection who were undergoing BAL for clinically suspected PCP while receiving prophylaxis with aerosolized pentamidine. All 34 consecutive cases in which BAL fluid was negative for $P$. carinii were prospectively followed for 3 months, and their clinical outcome was assessed.

The clinical suspicion of PCP was based on certain factors: immunodeficiency, respiratory symptoms (cough and dyspnea), fever, and/or evidence of infiltrates on a chest roentgenogram. Dyspnea was defined as shortness of breath with or without exertion, as reported by the patient. Fever was defined as a temperature of $>37.8^{\circ} \mathrm{C}$. All patients had received pentamidine ( $300 \mathrm{mg}$ every 4 weeks, with use of the Respirgard II [Marquest Medical Products, Englewood, $\mathrm{CO}]$ ) for at least 2 months before BAL was performed.

Fiberoptic bronchoscopy was performed in a standardized way: the fiberoptic bronchoscope was advanced to the wedge position in the middle lobe or the lingula pulmonis sinistri, and four $50-\mathrm{mL}$ boluses of $0.9 \% \mathrm{NaCl}$ were sequentially instilled and withdrawn; the recovery of fluid ranged from $50 \%$ to $80 \%$. In cases with localized pulmonary involvement, la- 
vage was performed on multiple lung segments, including those with infiltrates detected by roentgenography.

Transbronchial biopsy was not performed for any patient. The BAL fluid was examined for $P$. carinii with use of Giemsa and toluidine blue $O$ stains as well as fluoresceinconjugated monoclonal antibodies. Gram stains, stains for acid-fast bacteria, and cultures for bacteria, fungi, mycobacteria, and cytomegalovirus were also routinely done.

For all subjects, a chest roentgenogram was obtained within 1 week prior to BAL; the roentgenogram was evaluated by staff radiologists and later verified by a pulmonologist. The serum lactate dehydrogenase (LDH) level (normal values, $230-460 \mathrm{U} / \mathrm{L}$ ) and arterial blood gas were assessed within 1 week of BAL, and the CD4 lymphocyte count, within 2 months. The alveolar-arterial oxygen tension gradient $\mathrm{P}(\mathrm{A}-\mathrm{a}) \mathrm{O}_{2}$ was calculated by the simplified alveolar gas equation; the normal value $(\mathrm{mm} \mathrm{Hg}$ ) was derived as follows: $($ age $[$ years $] / 2)+5$.

Bronchitis or pneumonia was diagnosed by means of (1) bronchoscopic findings such as erythema or purulence in the airways, (2) exclusion of the presence of $P$. carinii, mycobacteria, or other specific pathogens, and (3) radiological evidence of newly developed infiltrates (in cases of pneumonia).

A treatment active against PCP was defined as the administration for at least 1 week of any dose of trimethoprim-sulfamethoxazole, dapsone, aerosolized or iv pentamidine (daily), or antitoxoplasmic agents. The patients continued the prophylaxis with aerosolized pentamidine ( $300 \mathrm{mg}$ every 4 weeks) after BAL was performed.

To assess how many cases would have qualified for a presumptive diagnosis of PCP if no BAL had been done, we determined which cases fulfilled the following four criteria [9]: (1) a history of dyspnea with exertion or a nonproductive cough of recent onset (within the past 3 months); (2) evidence on a chest roentgenogram of diffuse bilateral interstitial infiltrates; (3) demonstration by arterial blood gas analysis of an arterial $\mathrm{PO}_{2}$ of $<70 \mathrm{~mm} \mathrm{Hg}$ or an increase in the $\mathrm{P}(\mathrm{A}-\mathrm{a}) \mathrm{O}_{2}$; and (4) no evidence of bacterial pneumonia.

\section{Results}

During the study period of 19 months, 122 cases of PCP were diagnosed at our institution on the basis of examination of sputum or induced sputum (65 cases), BAL fluid (56), or pleural aspirate (1). In an additional 65 instances, BAL was done for suspected PCP but did not reveal $P$. carinii. Within this group, 33 patients ( 34 episodes) were receiving prophylaxis with aerosolized pentamidine; the study population comprised these 33 patients who had 34 cases of clinically suspected PCP.

The mean age of these patients ( 22 men and 11 women) was 35.7 years (range, 23-57 years). Seventeen were homosexuals, 10 were parenteral drug abusers, and six had no
Table 1. Clinical presentation, findings on a roentgenogram of the chest, and laboratory values before performance of BAL $(n=34)$.

\begin{tabular}{lc}
\hline Characteristic & No. (\%) of patients \\
\hline Fever & $28(82)$ \\
Cough & $29(85)$ \\
Dyspnea & $17(50)$ \\
Fever, cough, and dyspnea & $12(35)$ \\
Findings on chest roentgenogram $(n=33):$ & \\
$\quad$ Diffuse infiltrates & $19(58)$ \\
Lobar infiltrates & $8(24)$ \\
$\quad$ No abnormalities & $6(18)$ \\
Elevated LDH level $(>460 \mathrm{U} / \mathrm{L})(n=33)$ & $17(52)$ \\
Po ${ }_{2}$ of $<70$ mm Hg or elevated $\mathrm{P}(\mathrm{A}-\mathrm{a}) \mathrm{O}_{2}(n=12)$ & $7(58)$ \\
\hline
\end{tabular}

NOTE. LDH = lactate dehydrogenase in serum.

identified risk factor for HIV infection. In all 34 cases AIDS was diagnosed before BAL was performed. Thirty patients had previously had PCP and were receiving secondary pentamidine prophylaxis, while four were given primary prophylaxis. All were severely immunodeficient (the CD4 lymphocyte count was $<100 / \mathrm{mm}^{3}$ in 30 cases and $<200 / \mathrm{mm}^{3}$ in all cases). Treatment was given with zidovudine in 26 cases and with didanosine in three, while no antiretroviral treatment was given in five cases. Symptoms, laboratory data, and roentgenographic findings were compatible with the clinical suspicion of PCP (table 1). The median duration of symptoms before BAL was 3.4 weeks (range, 0-8 weeks).

Based on the results of BAL, a diagnosis was made in 28 cases (table 2). No diagnosis could initially be established in the remaining six cases; two of these patients were empirically treated for PCP despite the fact that $P$. carinii was not identified in the BAL fluid. One of these patients received dapsone $(100 \mathrm{mg} / \mathrm{d})$, trimethoprim $(900 \mathrm{mg} / \mathrm{d})$, and antibiotics because of concurrent Staphylococcus aureus pneumonia and was cured. The other received trimethoprimsulfamethoxazole $(75 / 15 \mathrm{mg} /[\mathrm{kg} \cdot \mathrm{d}])$, and his condition improved temporarily. Subsequently, however, he became febrile again and died after $2 \frac{1}{2}$ months; the autopsy revealed generalized toxoplasmosis but no PCP. In the other 32 cases, no treatment active against $P$. carinii was given, and none of the patients developed PCP during the 3-month follow-up period. Therefore, the negative predictive value of BAL was at least $94 \%$ ( 32 of 34 cases) if the two patients who were treated for PCP were considered as possibly (although not evidently) having undiagnosed PCP.

The final diagnoses in the 32 cases in which no treatment active against $P$. carinii was given included bronchitis (3 cases), pneumonia (18), nontuberculous mycobacteriosis (7), Kaposi's sarcoma (4, of which 3 were concurrent with pneumonia), and cerebral toxoplasmosis, cytomegalovirus pneumonitis, and nonspecific interstitial pneumonitis (1 each). Nontuberculous mycobacteriosis was detected by cul- 
Table 2. Diagnoses made at the time of BAL and at the end of the 3-month follow-up period.

\begin{tabular}{|c|c|}
\hline $\begin{array}{l}\text { Diagnosis at the time of BAL } \\
\text { (no. of patients) }\end{array}$ & $\begin{array}{l}\text { New diagnosis or clinical } \\
\text { aspects noted after follow-up } \\
\text { (no. of patients) }\end{array}$ \\
\hline $\begin{array}{l}\text { Bronchitis or pneumonia }(21)^{*} \\
\text { Kaposi's sarcoma (4)* } \\
\text { Nontuberculous mycobacteriosis }(4)^{\dagger} \\
\text { Cytomegalovirus pneumonitis (1) } \\
\text { Nonspecific interstitial pneumonitis (1) } \\
\text { Unclear (6) }\end{array}$ & $\begin{array}{l}\text { None } \\
\text { None } \\
\text { None } \\
\text { None } \\
\text { None } \\
\text { Therapy for PCP given; clinical } \\
\text { improvement (1 })^{\ddagger 8} \\
\text { Therapy for PCP given; death } \\
\text { after } 21 / 2 \text { months; autopsy } \\
\text { revealed generalized } \\
\text { toxoplasmosis }(1)^{8} \\
\text { Cerebral toxoplasmosis (1) } \\
\text { Nontuberculous } \\
\text { mycobacteriosis }(3)^{\dagger}\end{array}$ \\
\hline
\end{tabular}

* In three cases both pneumonia and Kaposi's sarcoma were diagnosed.

t These cases of mycobacterial infection were due to $M$. avium complex (4), M. kansasii (1), M. malmoense (1), and both $M$. avium complex and $M$. kansasii (1); all cases were confirmed by cultural detection of the pathogens in specimens of the blood or from other organs.

"Initial findings were reported as "possibly few cysts of $P$. carinii," but no cysts were seen in a later review; concurrent pneumonia due to Staphylococcus aureus was treated with additional antibiotics.

These two patients were empirically treated for PCP and regarded as possibly having undiagnosed PCP.

ture in 3 of 6 cases in which no acid-fast bacilli were detected in BAL fluid and the etiology was initially unclear. The slow cultural growth of nontuberculous mycobacteria was thus an important factor contributing to the delayed diagnosis for these patients.

Elevation of serum LDH occurred in all disease categories: $9(43 \%)$ of 21 cases of bronchitis or pneumonia; $3(43 \%)$ of 7 cases of nontuberculous mycobacteriosis; and $2(50 \%)$ of 4 cases of Kaposi's sarcoma.

Four patients died during follow-up. An autopsy was performed on three (including the patient with generalized toxoplasmosis) and revealed no evidence of PCP. The fourth patient had been treated for bacterial pneumonia following BAL and recovered clinically but died 2 months later of an unknown cause.

\section{Discussion}

In this prospective study of patients evaluated for suspected PCP while receiving pentamidine prophylaxis, the negative predictive value of BAL was at least $94 \%$. Although all patients in this series were at high risk for PCP [10-12] because of previously diagnosed AIDS and severe immunodeficiency, as well as their symptoms and the clinical and laboratory findings (table 1), no cases of undiagnosed PCP were positively identified during the follow-up. The decision to treat two patients for PCP despite the negativity of BAL fluid for $P$. carinii was made by their physicians; these patients were considered as possibly having undiagnosed PCP despite the fact that pneumonia of a different etiology might have responded to the treatment. Our study confirms the finding that aerosolized pentamidine does not substantially decrease the diagnostic yield of BAL in detection of $P$. carinii [13]. Previous retrospective studies of small numbers of patients, in which yields of only $50 \%-62 \%$ were reported $[7,8]$, directly compared results of examinations of BAL and transbronchial biopsy specimens but did not use fluorescein-conjugated monoclonal antibodies (which may increase the sensitivity of BAL [14]). Comparisons of the morphologic detection of $P$. carinii and the subsequent development of disease, as made in our study, are limited by the difference in study designs. However, the negative predictive value of BAL was validated by our patients' clinical course, which supports the practice of employing BAL without transbronchial biopsy in all cases in which spontaneous or induced sputum cannot be obtained or does not allow a definite diagnosis. Transbronchial biopsy employed in addition to BAL would implicate an unnecessary risk of pneumothorax and bleeding $[15,16]$ and should remain a second-step diagnostic tool in cases requiring further evaluation.

The decision to assess the clinical outcome 3 months after BAL for development of PCP was based on the natural history of PCP [17]; we assumed that untreated PCP would further progress within this time. For patients who received no prophylaxis for PCP, even a shorter follow-up (6 weeks) enabled us to differentiate between PCP and other respiratory conditions [12].

When the results of screening with BAL were disregarded and only the symptoms and signs noted before BAL were considered, four patients (12\%) met the criteria for presumptive diagnosis of PCP [9]. Without performance of BAL, these cases would have been misdiagnosed as PCP and the patients would have been unnecessarily treated. Among patients in every disease category, elevation of serum LDH was found in $43 \%-50 \%$, thus confirming that $\mathrm{LDH}$ is a sensitive [12] but rather nonspecific predictor of PCP. These findings illustrate the limitations of algorithms designed to predict PCP [12] and emphasize the value of the detection of $P$. carinii during the diagnostic process. Because of the high toxicity of the agents presently used in treatment regimens for PCP [18, 19], the avoidance of unnecessary therapy is important in maintaining the patients' quality of life.

Before BAL was performed, we were able to obtain spontaneous or induced sputum from 22 of the patients studied. The performance of BAL for these patients was indicated by the finding that the sputum was contaminated or nondiagnostic, thereby necessitating further diagnostic efforts. Despite its slightly lower sensitivity for detection of PCP, examination of induced sputum should nevertheless represent the 
first diagnostic step for immunodeficient patients with respiratory symptoms.

Our study shows that clinical findings characteristic of PCP can originate from miscellaneous diseases, including bacterial infections of the lower respiratory tract (62\% of all cases). Haemophilus influenzae, S. aureus, and pneumococci were among the bacterial pathogens isolated in our study and should be considered with respect to not only the diagnostic workup but also the choice of antibiotics for HIV-infected patients with respiratory infections [20]. Nontuberculous mycobacteriosis and Kaposi's sarcoma accounted for $21 \%$ and $12 \%$ of cases, respectively. While the proportion of cases of AIDS with tuberculosis or nontuberculous mycobacteriosis is increasing [21], the detection of these mycobacteria still causes a diagnostic delay because of their slow cultural growth; more-rapid detection methods such as polymerase chain reaction or chromatography are needed to enable initiation of adequate treatment as early as possible [22].

In summary, for HIV-infected patients with pulmonary symptoms who are at risk for PCP, detection of $P$. carinii should definitely be attempted; however, BAL fluid that is shown to contain no $P$. carinii during a period of prophylaxis with pentamidine is sufficiently reliable evidence for excluding a diagnosis of PCP. A vigorous search for other causes of pulmonary disease is required for such patients, as an exact diagnosis is necessary for instituting adequate therapeutic measures for the wide spectrum of possible diseases.

\section{References}

1. Girard PM, Landman R, Gaudebout C, et al. Prevention of Pneumocystis carinii pneumonia relapse by pentamidine aerosol in zidovudinetreated AIDS patients. Lancet 1989;1:1348-53.

2. Leoung GS, Feigal DW Jr, Montgomery AB, et al. Aerosolized pentamidine for prophylaxis against Pneumocystis carinii pneumonia: the San Francisco community prophylaxis trial. N Engl J Med 1990;323:769-75.

3. Hirschel B, Lazzarin A, Chopard P, et al. A controlled study of inhaled pentamidine for primary prevention of Pneumocystis carinii pneumonia. N Engl J Med 1991;324:1079-83.

4. Kales CP, Murren JR, Torres RA, Crocco JA. Early predictors of inhospital mortality for Pneumocystis carinii pneumonia in the acquired immunodeficiency syndrome. Arch Intern Med 1987;147: 1413-7.

5. Broaddus C, Dake MD, Stulbarg MS, et al. Bronchoalveolar lavage and transbronchial biopsy for the diagnosis of pulmonary infections in the acquired immunodeficiency syndrome. Ann Intern Med 1985; 102:747-52.

6. Golden JA, Hollander H, Stulbarg MS, Gamsu G. Bronchoalveolar lavage as the exclusive diagnostic modality for Pneumocystis carinii pneumonia: a prospective study among patients with acquired immunodeficiency syndrome. Chest 1986;90:18-22.
7. Jules-Elysee KM, Stover DE, Zaman MB, Bernard EM, White DA. Aerosolized pentamidine: effect on diagnosis and presentation of Pneumocystis carinii pneumonia. Ann Intern Med 1990;112:750-7.

8. Edelstein H, McCabe RE. Atypical presentations of Pneumocystis carinii pneumonia in patients receiving inhaled pentamidine prophylaxis. Chest 1990;98:1366-9.

9. World Health Organization. Acquired immunodeficiency syndrome (AIDS). 1987 revision of CDC/WHO case definition for AIDS. Wkly Epidemiol Rec 1988;63:1-8.

10. Masur H, Ognibene FP, Yarchoan R, et al. CD4 counts as predictors of opportunistic pneumonias in human immunodeficiency virus (HIV) infection. Ann Intern Med 1989;111:223-31.

11. Phair J, Munoz A, Detels R, et al. The risk of Pneumocystis carinii pneumonia among men infected with human immunodeficiency virus type 1. N Engl J Med 1990;322:161-5.

12. Katz MH, Baron RB, Grady D. Risk stratification of ambulatory patients suspected of Pneumocystis pneumonia. Arch Intern Med 1991;151:105-10.

13. Geaghan S, Fahey J, McGinty E, et al. Impact of prophylactic aerosolized pentamidine on the laboratory diagnosis of Pneumocystis carinii pneumonia [abstract no 850]. In: Program and abstracts of the 30th Interscience Conference on Antimicrobial Agents and Chemotherapy (Atlanta). Washington, DC: American Society for Microbiology, 1990:228.

14. Elvin KM, Björkman A, Linder E, Heurlin N, Hjerpe A. Pneumocystis carinii pneumonia: detection of parasites in sputum and bronchoalveolar lavage fluid by monoclonal antibodies. BMJ 1988;297:3814.

15. Fulkerson WJ. Fiberoptic bronchoscopy. N Engl J Med 1984; 311:511-5.

16. Flick G, Barbers R, Gong H. Bedside fiberoptic bronchoscopy and bronchoalveolar lavage (BAL) for the diagnosis of Pneumocystis carinii pneumonia in patients with the acquired immunodeficiency syndrome (AIDS) [abstract]. Am Rev Respir Dis 1985;131 (suppl):A221.

17. Kovacs JA, Hiemenz JW, Macher AM, et al. Pneumocystis carinii pneumonia: a comparison between patients with the acquired immunodeficiency syndrome and patients with other immunodeficiencies. Ann Intern Med 1984; 100:663-71.

18. Medina I, Mills J, Leoung G, et al. Oral therapy for Pneumocystis carinii pneumonia in the acquired immunodeficiency syndrome: a controlled trial of trimethoprim-sulfamethoxazole versus trimethoprimdapsone. N Engl J Med 1990;323:776-82.

19. Klein NC, Duncanson FP, Lenox TH, et al. Trimethoprim-sulfamethoxazole versus pentamidine for Pneumocystis carinii pneumonia in AIDS patients: results of a large prospective randomized treatment trial. AIDS 1992;6:301-5.

20. Polsky B, Gold JWM, Whimbey E, et al. Bacterial pneumonia in patients with the acquired immunodeficiency syndrome. Ann Intern Med 1986;104:38-41.

21. Schwartländer B, Horsburgh CR Jr, Hamouda O, Skarabis H, Koch MA. Changes in the spectrum of AIDS-defining conditions and decrease in CD4+ lymphocyte counts at AIDS manifestation in Germany from 1986 to 1991. AIDS 1992;6:413-20.

22. American Thoracic Society. Diagnosis and treatment of disease caused by nontuberculous mycobacteria. Am Rev Respir Dis 1990; 142:940-53. 